tenderness was felt in 16 , but none was considered to have evidence of early abscess formation and all were allowed home. Three patients, not among the 16 , later returned with pelvic abscesses.

\section{Discussion}

Children find a rectal examination unpleasant and over a third experienced severe discomfort in this study. This is acceptable only if essential information affecting management is obtained. In this series, the diagnosis of acute appendicitis in over $90 \%$ of cases was made from the clinical history and abdominal examination. In two cases the rectal tenderness indicated the correct diagnosis. Rectal tenderness was present in $12 \%$ of children who did not have acute appendicitis. It seems reasonable, therefore, to omit routine rectal examination in children in whom the diagnosis of acute appendicitis is obvious from the history and abdominal signs. It remains essential, however, in those with acute abdominal symptoms but with inconclusive or no abdominal signs. Pelvic appendicitis and abscess may yield very few abdominal signs. Our results show that the examination on discharge home seems to be a fruitless exercise; in no patient was any information gained that affected the decision already made that the child was fit to leave hospital.

We conclude from this study that:

(1) Rectal examination is unpleasant for children;

(2) The diagnosis of acute appendicitis in children may be made in over $90 \%$ of cases without rectal examination;

(3) When the diagnosis is uncertain a rectal examination is sometimes useful to diagnose pelvic appendicitis or other pelvic pathology;

(4) Rectal examination is a very poor early indicator of pelvic sepsis after appendicectomy.

Correspondence to Mr A P Dickson, Surgical Paediatric Department, Western General Hospital, Edinburgh EH4 2XU.

Received 22 March 1985

\title{
Intravenous immunoglobulin for neonatal isoimmune thrombocytopenia
}

\author{
M DERYCKE, M DREYFUS, J C ROPERT, AND G TCHERNIA \\ Departments of Haematology and Neonatology, Hôpital Antoine Béclère, Clamart, France
}

SUMMARY An infant with severe, isoimmune thrombocytopenia secondary to maternal anti-Pla 1 immunisation was treated successfully by intravenous gammaglobulin $(400 \mathrm{mg} / \mathrm{kg}$ per day for five days). This treatment compared with Pla 1 negative platelet transfusions is discussed.

Materno-fetal incompatibilities in the Pla 1 system may lead to isoimmune neonatal thrombocytopenia and severe bleeding either in utero, during delivery, or in the first days of life. This condition is associated with a high risk of neonatal mortality or neurological sequelae secondary to intracranial haemorrhage.

Apart from performing a caesarean section to prevent obstetrical trauma, no preventive treatment has been available until recently. Exchange transfusions or transfusions with Pla 1 negative platelets in cases of severe postnatal bleeding or as a preventative measure have provided only temporary benefit. ${ }^{1}$ Neonatal thrombocytopenia, however, will resolve spontaneously over a period of three to four weeks, indicating the progressive decrease of maternal antibodies in the infant's circulation.

In over $50 \%$ of children with idiopathic thrombocytopenic purpura high dose intravenous gammaglobulin has been shown to increase the platelet count to normal values within a short period. This treatment has subsequently been used in neonatal thrombocytopenia related to maternal idiopathic thrombocytopenic purpura.

We report an infant with isoimmune neonatal thrombocytopenia treated with intravenous gammaglobulin. ${ }^{2}$

\section{Case report}

A 2 hour old boy weighing $2350 \mathrm{~g}$ was referred to our hospital with thrombocytopenia. He was the second child of a 23 year old woman, whose 38 week pregnancy, labour, and delivery had been uncomplicated. The mother's platelet count was normal at delivery. Her first born infant, a girl, had been thrombocytopenic at birth; this had been attributed 
to an incompatibility in the Pla 1 system and had resolved without any treatment or sequelae. Despite this medical history, our patient was delivered vaginally. Physical examination of the infant showed large bruises on the face as well as diffuse petechiae. His Apgar score was 10 and neurological examination was normal. Platelet count in a cord blood sample was $6 \times 10^{9} / 1$. Four hours after birth the haemoglobin concentration was $16.4 \mathrm{~g} / \mathrm{dl}$; white blood count was $20.9 \times 10^{9} / 1$ with $60 \%$ leucocytes, $10 \%$ band cells, $25 \%$ lymphocytes, $5 \%$ monocytes, and $2 \%$ normoblasts; and platelets were $30 \times 10^{9} / 1$. Coagulation studies were normal with fibrinogen $220 \mathrm{mg} / 100 \mathrm{ml}$, activated partial thromboplastin time $64 / 40$, prothrombin time $60 \%$, and thrombin time 26/15. Direct Coombs's test performed on newborn red blood cells was negative. The bacteriological data did not show any evidence of infection, and echoencephalography was also normal.

The infant's subsequent clinical course was uncomplicated. There was a moderate jaundice on day 3 but no visceral, cerebral, or ocular bleeding was noted. On day 4 , the platelet count was $14 \times 10^{9} / 1$, and an infusion of $\mathrm{pH} 4$ treated immunoglobulin (Sandoglobulins, Basel, in 3\% saline solution) was started at a dose of $400 \mathrm{mg} / \mathrm{kg}$ per day, from day 4 to day 8 (five days). Infusions were performed over a three hour period each day and were well tolerated. Correction of thrombocytopenia was noted between day 8 and day 10 , when the platelet count was $180 \times 10^{9} / 1$ (Figure). The patient was discharged on day 13; regular follow up during the next two months showed that the platelet count remained within the normal range.

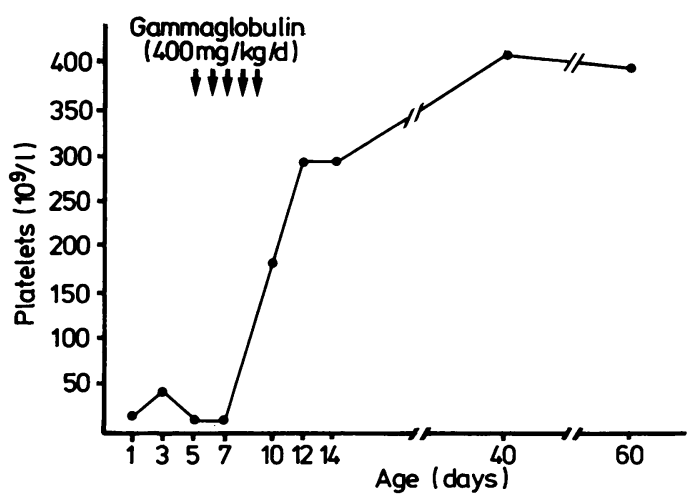

Figure Treatment of neonatal, isoimmune thrombocytopenia by high dose intravenous gammaglobulin.

\section{Immunological results}

The mother's platelets were Pla 1 negative while the father's and child's platelets were found to be Pla 1 positive. The presence of anti-Pla 1 isoimmune antibodies in the maternal serum was assayed by a radioimmunological Coombs's test using a panel of platelets. ${ }^{3}$ The test was positive for the father's platelets, as well as for platelets from two other Pla 1 positive control subjects. Control with either autologous maternal platelets, or platelets from a Pla 1 negative control subject were negative. The maternal serum, however, reacted positively with one other Pla 1 negative control, due to the presence of anti-HLA antibodies. All the tests were performed again on platelets treated with chloroquine, and were found positive with the Pla 1 positive platelets, showing that the anti-platelet antibodies did not belong exclusively to HLA antibodies. The direct Coombs's test was not performed for the infant, due to the insufficient sample size, and was found to be negative after treatment.

\section{Discussion}

The mechanism by which intravenous gammaglobulin resolves idiopathic thrombocytopenic purpura is still controversial. The two most commonly admitted hypotheses being:

(a) A decrease in platelet binding of gammaglobulin or immune complexes, due to the competition between infused gammaglobulin and the immune antibodies on the platelet crystallisable fragment (Fc) receptor, ${ }^{4}$

(b) A binding of infused gammaglobulin to the crystallisable fragment receptor of the macrophages which reduces their effectiveness in phagocytosing the gammaglobulin sensitised platelets.

Compared with other currently available treatments in isoimmune neonatal thrombocytopenia, intravenous gammaglobulin treatment has the following advantages:

(1) It may be easily initiated, immediately after birth, and seems to be well tolerated in neonates as well as in children and adults. pH4 treatment of globulin eliminates completely viruses such as lymphadenopathy associated virus or hepatitis B virus, and no transmission of infectious disease has yet been documented with Sandoglobulins.

(2) The resolution of thrombocytopenia is usually rapid, starting 24 to 48 hours after starting treatment (but in our case the response was moderately delayed) and is sustainable for a period longer than a week. We cannot, however, assume that it was not a spontaneous resolution, and further similar treatments will allow us to evaluate a response rate. 
The effective resolution of thrombocytopenia in neonates with isoimmune $\mathrm{Pla} 1$ thrombocytopenia suggests that this mode of treatment could be used in utero. When the diagnosis is suggested by previous family medical history, negative Pla 1 platelets in the mother and low platelet counts on fetal blood samples, intravenous gammaglobulin infused in the fetus could be an alternative to transfusion of $\mathrm{Pla} 1$ negative platelets in utero. ${ }^{5}$

\author{
References \\ ${ }^{1}$ Sitarz AL, Driscoll JM, Wolff JA. Management of isoimmune
} thrombocytopenia. Am J Obstet Gynecol 1976;124:39-42.

\footnotetext{
2 Sidiropoulos D, Straume B. The treatment of neonatal isoimmune thrombocytopenia with intravenous immunoglobulins. Blut 1984;48:383-6.

${ }^{3}$ Soulier JP, Patereau C, Drouet J. Platelet indirect radio-active Coombs test: its utilization for Pla 1 grouping. Vox Sang 1975;29:253-68.

${ }^{4}$ Imbach P, Jungi TW. Possible mechanisms of intravenous immunoglobulins treatment in childhood ITP. Blut 1983;46: 117-24.

5 Daffos F, Forestier F, Muller JY, et al. Prenatal treatment of allo-immune thrombocytopenia. Lancet 1984;ii:632.
}

Correspondence to Dr M Derycke, Laboratoire d'Hématologie, Hôpital Antoine Béclère 157, Avenue de la Porte de Trivaux, 92140 Clamart, France.

Received 13 March 1985

\title{
Inguinal hernias are common in preterm infants
}

\author{
G R BOOCOCK AND P J TODD
}

Alder Hey Children's Hospital and Royal Liverpool Children's Hospital, Liverpool

SUMmaRY The incidence of inguinal hernia was compared in term and preterm infants. The risk was considerably higher in the preterm group in both sexes. No particular complications of preterm birth were found to predispose to inguinal hernia.

Surgical texts indicate an increased risk of inguinal hernia in preterm infants. Increasing survival of these infants has made this a common problem on neonatal units. The risks of hernia complications in a frail infant are clear and we felt that a more detailed study of the extent of the problem and an attempt to define possible predisposing factors would be a useful exercise.

\section{Method}

The population of Liverpool is served by three maternity units and two children's hospitals where all surgery is performed. Names of all infants with Liverpool addresses born in 1981 who had hernia operations under the age of 6 months were obtained from surgical registers. Birthweight and gestational age were taken from the neonatal records. The notes of those born before 36 weeks' gestation were studied to discover any complications of prematurity which may have predisposed them to the development of inguinal hernia. The incidence of right, left, and bilateral hernias was compared for boys and girls of different gestational ages.

\section{Results}

Sixty five patients were identified, of whom 25 were born before 36 weeks' gestation; 15 of these were of very low birthweight, that is less than $1500 \mathrm{~g}$. As expected, there was a noticeable sex difference with an overall $8: 1$ predominance of boys. The ratio was higher in the preterm group (12:1) but the difference between term and preterm groups did not reach statistical significance.

Right sided hernias predominated in term boys and bilateral hernias in term girls. The preterm group had a preponderance of bilateral hernias in both sexes but the number of girls was small. Left inguinal hernia alone was unusual.

The risk of a child developing a hernia and requiring operation before 6 months of age was calculated. Relative risks in the different gestational groups and the very low birthweight group are shown in the Table.

Table Percentage risk of developing inguinal hernia under the age of 6 months in boys and girls at different gestational ages and weights

\begin{tabular}{cllll}
\hline & & No of births & Hernias & $\%$ Risk \\
\hline Gestational age & Boys & 5649 & 35 & $0 \cdot 6$ \\
$>36$ wks & Girls & 5299 & 5 & $0 \cdot 09$ \\
Gestational age & Boys & 326 & 23 & 7 \\
$<36$ wks & Girls & 302 & 3 & 1 \\
Weight & Boys & 91 & 13 & 14 \\
$<1500 \mathrm{~g}$ & Girls & 81 & 2 & $2 \cdot 5$ \\
\hline
\end{tabular}

